\title{
Date Range
}

National Cancer Institute

\section{Source}

National Cancer Institute. Date Range. NCI Thesaurus. Code C93545.

The date and time span specifying when an activity or event began and ended. 